are founding members of YIBcap and former D. J. Cohen Fellows, but have no financial or other conflicts of interest that might bias their work.

\section{References}

Adler, R., Martin, A., Park, C., et al (2007) Mentoring young researchers: can the Donald I Cohen Fellowships model be applicable and useful to Australasian psychiatry? Australasian Psychiatry, 15, 232-236.
Ehrlich, S. \& Stegemann, T. (2007) [Young Investigators in Biological child and adolescent psychiatry (YIBcap) - insights after one year of networking.] Zeitschrift für Kinder- und Jugendpsychiatrie und Psychotherapie, 35, 59-63.

Fegert, J. M. \& Vitiello, B. (2007) Peer-reviewed, high quality, worldwide information on all topics relevant to child and adolescent mental health. Child Adolescent Psychiatry and Mental Health, 1, 1.

Martin, A. (2005) Ignition sequence: on mentorship. Journal of the American Academy of Child and Adolescent Psychiatry, 44, 1225-1229.

Shortt, S. E. (2004) Making sense of social capital, health and policy. Health Policy, 70, 11-22.

\title{
Child and adolescent mental health services in Pakistan: current situation, future directions and possible solutions
}

\author{
Faria Khan, ${ }^{1}$ R. K. Shehzad ${ }^{2}$ and Haroon R. Chaudhry ${ }^{3}$
}

${ }^{1}$ Child and Adolescent Services for Early Intervention, Lancashirecare NHS Trust, Lancashire, UK 2Department of Paediatrics, Clonmel General Hospital, Clonmel, Ireland ${ }^{3}$ Department of Psychiatry, Fatima Jinnah Medical College, Lahore, Pakistan

W orldwide, mental disorders are on the increase (Gadit, 2007) and an estimated $10-20 \%$ of children have one or more mental or behavioural problems (Park, 2002). There is an urgent and serious need to pay attention to the mental health needs of children in low- and middle-income countries (Rahman et al, 2000). The initial survey for the World Health Organization's Atlas project (Sherer, 2002) showed that $41 \%$ of countries surveyed had no mental health policy and $28 \%$ had no separate budget for mental health.

In Pakistan, with a population of 169 million, the allocation to adult mental health is hardly $1 \%$ of the annual health budget, and there is no specific allocation for children and adolescents. The provision of child and adolescent mental health services is very fragmented nationally.

This paper highlights the status of child and adolescent psychiatry services in Pakistan and makes some suggestions on how to develop them within the available resources.

\section{Current situation}

The current situation in Pakistan is that there is no formal referral pathway to psychiatric services for children. Children with mental and behavioural problems present to general practitioners in the community or to psychiatric out-patient departments, where they are seen by general adult psychiatrists, most of whom have little if any formal training in child psychiatry. However, it would not be improper to hypothesise that children reach these services after attending spiritual and faith healers.

The more common referrals involve neuropsychiatric and neurodevelopmental disorders, including behavioural sequelae of epilepsy and brain injury, pervasive developmental disorders, attention-deficit hyperactivity disorder, Tourette syndrome, early-onset psychosis, other psychoses, psychosomatic/dissociative disorders, eating disorders, depression, anxiety and obsessive-compulsive disorders.

If children require admission, they stay on general adult psychiatric wards with adults who are acutely mentally unwell. The staff have very little formal training in managing children. Hence, the burden falls on the parents and carers to look after and manage them, leading to further anxiety and distress, especially if a parent has to take time off work.

\section{Future directions and possible solutions}

Developing paediatric-psychiatric liaison services

In Pakistan, in order to make use of scarce resources and still provide an appropriate in-patient setting, a suitable model could be for admissions to be to the paediatric ward, but with full support from the psychiatric team. Each adult psychiatry department could set up a paediatric liaison facility. Where the child is acutely disturbed and is difficult to manage in the paediatric ward, there could be a designated area on the psychiatric ward, preferably away from adult patients.

Lack of communication among existing paediatric and psychiatric specialties needs to be addressed through integration. One way to establish services would be to hold joint departmental meetings once a month, to which ward nurses, psychologists and local general practitioners should also be invited, to increase their awareness of psychiatric disorders in children. Attendance at paediatric ward rounds and joint teaching sessions, journal clubs, case presentations and so on would allow a clearer understanding of the issues faced by all involved. 


\section{Establishing multidisciplinary teams}

In child psychiatry, an ideal team consists of professionals from different disciplines, including psychiatrists, psychologists, occupational therapists, nursing staff, family therapists, teachers and psychotherapists. There are usually close links with paediatricians and local social services.

In Pakistan, it is difficult but not impossible to develop a multidisciplinary team. Currently, teams consist mainly of psychiatrists, psychologists and nurses. Many units have occupational therapy as part of the in-patient experience and this is an area that could be developed in a more structured way. Psychiatrists working in adult psychiatric units attached to a general hospital (which is now the usual arrangement) often have good links with teams in the rest of the hospital and frequently receive and send referrals to them. Similar links could be developed with local paediatricians, where a child psychiatry service is to be developed.

\section{Foreign aid}

Given the shortage of specialist child psychiatry services, one way forward could be for child and adolescent psychiatrists trained abroad to join with local psychiatrists. Working as a team, they could develop culture-specific guidelines. This would be especially helpful if there were clear protocols for the management of various mental health problems in children. Currently this is happening in an ad hoc way (Rahman et al, 2000). The help of other organisations could be enlisted in achieving this; for example, the Royal College of Psychiatrists has its Volunteer Programme for Senior Psychiatrists and Specialist Registrars, which aims to match senior professionals working in the UK with departments in different parts of the world looking to develop expertise in particular areas (see http://www.rcpsych.ac.uk). The South Asian Forum for Psychiatry provides training in various countries; this will also prove to be a useful resource in providing training in child and adolescent mental health services.

Similarly, the Higher Education Commission of Pakistan welcomes foreign-trained professionals who are willing to contribute to service development in the country.

Another example worth mentioning is DRIP - Disaster Relief by Irish and Pakistani doctors (see http://www. dripireland.org). A voluntary organisation of Pakistani-origin doctors living in Ireland, DRIP is developing emergency medical services in Pakistan and extending its input to all branches of medicine with a view to introducing culturally appropriate protocols and guidelines in all the fields of medicine. Local and foreign experts will provide a platform for future developments in the field. DRIP has recently established an adult and child psychiatry branch.

\section{Training of health professionals}

Medical training at undergraduate and postgraduate levels needs both to create awareness and to stress the importance of child and adolescent psychiatry.

Joint problem-based training sessions with paediatricians and psychiatrists needs to be rolled out in teaching set-ups, with a view to developing liaison services.

Nurses need to be trained in the management of children with psychiatric disorders. They should develop counselling skills and be able to run structured and unstructured groups. These skills can be imparted to them during their training years by psychologists and psychiatrists.
There is also a question of training general practitioners (GPs). The aim would be to increase their awareness of protocols and referral pathways. It should be made mandatory for GPs to do 6 months of psychiatry to obtain their licence. Currently, 1-week programmes in psychiatry for GPs are conducted at Fountain House, Lahore, which is affiliated with the World Psychiatric Association's section on education (these contribute to the GPs' programme of continuing medical education).

In Pakistan mental health professionals need first to identify their area of special interest in treatment modalities, such as cognitive-behavioural therapy, family therapy, psychotherapy, education or occupational therapy. They then need to develop their interests by pursuing the appropriate training. The Royal College of Psychiatrists is looking to offer short periods of training to appropriately qualified individuals.

In hospitals, heads of departments have been organising training programmes and inviting local and foreign speakers, in this way keeping staff abreast of innovative and exciting developments in the field. Overseas Pakistani psychiatrists can be a useful source of training. At the Institute of Psychiatry at Rawalpindi General Hospital, email consultation and supervision with child psychiatrists in the UK has proved to be a very useful resource (Rahman et al, 2006). Similar set-ups could be developed in other parts of the country.

\section{Involvement of the community}

Discharge plans need careful consideration to ensure that all supports and follow-up plans are in place. In Pakistan the family and carers take on this role. Family psychoeducation for the early identification of symptoms of relapse is desirable, especially when families live in areas where they cannot easily come for regular follow-up. This information needs to be provided by the treating mental health professionals. Training/psychoeducation of the community is being carried out by mental health professionals in a number of areas; for instance, a programme in Lahore involving mothers, Imams (religious leaders) and teachers is running an awareness campaign on psychiatric disorders.

Given the importance of school in the lives of children, teacher training is another area to focus on. Several voluntary organisations, such as Amin Maktab and the National Society for Emotionally and Behaviourally Handicapped Children, have been running outreach programmes, training parents and local community volunteers.

\section{Research and audit}

There is a need to develop robust research projects to identify the prevalence of childhood mental illness. (Currently the lead author is conducting one such project in a teaching hospital in Lahore.) A summary of significant international epidemiological studies concluded that the rate and range of psychiatric symptoms in children is similar in lower- and higher-income countries (Fayyad et al, 2001). However, the prevalence of child mental health problems in Pakistan is higher than has been reported in studies from other countries (Syed et al, 2007a). Research in the area of understanding the psychopathology of mental disorders in children in our cultural context needs to be explored (Nikapota, 1991). Furthermore, 'needs assessment' of the target population is another area worth exploring. 


\section{Conclusion}

In Pakistan, the national annual budget for mental health is far below what would be necessary to meet the needs of the population. There are very few child mental health facilities in the country (Syed et al, 2007b).

Collaborative working between professionals managing children needs to be strengthened. Management guidelines have to be developed, as this will enable good clinical care and also facilitate research and audit. Research is needed to identify the prevalence, psychopathology and needs of mental health problems in children and priorities for management. Psychiatrists trained abroad could be a source of support and training, preferably on a longer-term basis, through email consultation and supervision. The transfer of skills through workshops and short training courses also is effective.

Children lacking in mental health have an adverse effect on the country's productivity, economic stability and viability. Recent research from Karachi, Pakistan, has highlighted effective models of utilisation of scarce child mental health resources within the current health and educational setup (Syed et al, 2007b). Policy-makers in the face of lack of finances and resources need to make decisions based on the efficacy of proposed and evaluated interventions which are not only beneficial to the population in question but also cost-effective.

\section{Acknowledgements}

The authors would like to thank Dr Amina Tareen, Consultant Child and Adolescent Psychiatrist (London, UK), for her expert comments and support in the writing of this paper.

\section{References}

Fayyad, J. A., Jahshan, C. S. \& Karam, E. G. (2001) Systems development of child mental health services in developing countries. Child and Adolescent Psychiatric Clinics of North America, 10, 745-762.

Gadit, A. A. (2007) Comparison between a developed and a developing country. Journal of Medical and Biological Sciences, 1, 1-7.

Nikapota, D. A. (1991) Child psychiatry in developing countries. British Journal of Psychiatry, 158, 743-751.

Park, K. M-E. (2002) Up to one fifth of the world's children has mental or behavioral problems. UN Chronicle, June-August. See http://www. un.org.pk/gmc/chronicle-bah-prob.htm (last accessed August 2008).

Rahman, A., Mubbashar, M., Harrington, R., et al (2000) Annotation. Developing child mental health services in developing countries. Journal of Child Psychology and Psychiatry and Allied Disciplines, 41, 539-546.

Rahman, A., Nizami, A., Minhas, A., et al (2006) E-mental health in Pakistan: a pilot study of training and supervision in child psychiatry using the internet. Psychiatric Bulletin, 30, 149-152.

Sherer, R. (2002) Mental health care in the developing world. Psychiatric Times, 19(1).

Syed, E. U., Hussein, S. A. \& Mahmud, S. (2007a) Screening for emotional and behavioural problems amongst 5-11-year-old school children in Karachi, Pakistan. Social Psychiatry and Psychiatric Epidemiology, 42, 421-427.

Syed, E. U., Hussein, S. A. \& Yousafzai, A. W. (2007b) Developing services with limited resources: establishing a CAMHS in Pakistan. Child and Adolescent Mental Health, 12, 121-124.

\section{Psychiatry in Belgium}

\section{Benjamin J. Baig ${ }^{1}$ and Veronique Delvenne ${ }^{2}$}

${ }^{1}$ Clinical Lecturer in Psychiatry, Division of Psychiatry, University of Edinburgh, Royal Edinburgh Hospital, Edinburgh EH10 5HF, UK, email bbaig@staffmail.ed.ac.uk

${ }^{2}$ Vice-Présidente, Société Belge Francophone de Psychiatrie et des Disciplines Associées de l'Enfance et de l'Adolescence, Professor of Child and Adolescent Psychiatry, Free University of Brussels, Espace Thérapeutique Enfants-Adolescents-Parents, 24 Rue Ketels, 1020 Brussels, Belgium, email v.delvenne@skynet.be

$$
T_{\mathrm{n}}^{\mathrm{he}}
$$
he Kingdom of Belgium is a high-income country in northern Europe with an approximate area of $33000 \mathrm{~km}^{2}$ and a population of 10.5 million. The proportion of the population under the age of 15 years is $17 \%$ and the proportion of the population above the age of 60 years is $22 \%$. Life expectancy at birth is 75.2 years for males and 81.5 years for females. As a founding member of what is now the European Union, it hosts the headquarters of the European Commission and the European Parliament, as well as other major organisations, including NATO.

Belgium is divided into the Flemish-speaking region of Flanders, in the north, with $58 \%$ of the population, the French-speaking southern region of Wallonia, inhabited by $32 \%$, and the Brussels capital region, officially bilingual, inhabited by $10 \%$ of the population. A small German-speaking community exists in eastern Wallonia. Belgium's linguistic diversity is reflected in the organisation of its psychiatric institutions and legislation.

\section{Mental health policy}

In Belgium, as both federal government and communities are in charge of different parts of the mental health service, there is both a national mental health policy, formulated in 1988 , and a community mental health policy. A substance misuse policy is present which was initially formulated in 1921 and a national mental health programme was formulated in 1990 and updated in 1999.

Belgium has a suicide prevention policy, implemented by the Mental Health Centre in Brussels. It focuses on the quality of help offered and on the efficacy of the services from the 